\title{
Consumo alimentar de magnésio em pacientes com síndrome metabólica em uso de hidroclorotiazida
}

\author{
Food intake of magnesium in patients with metabolic syndrome using hydrochlorothiazide
}

\section{DOI: $10.37111 /$ braspenj.2020353014}

Daniele dos Santos e Santos

Vera Ferreira Andrade de Almeida²

Luana de Oliveira Leite ${ }^{3}$

Edilene Maria Queiroz Araújo ${ }^{4}$

Fernando Luís de Queiroz Carvalho ${ }^{5}$

Domingos Lázaro Souza Rios ${ }^{6}$

\begin{abstract}
Unitermos:
Consumo de alimentos. Interações alimento-droga. Síndrome metabólica. Hipertensão. Hidroclorotiazida. Deficiência de Magnésio.
\end{abstract}

\section{Keywords:}

Food consumption. Food-drug interactions. Metabolic syndrome. Hypertension. Hydrochlorothiazide. Magnesium deficiency.

\section{Endereço para correspondência: \\ Luana de Oliveira Leite}

Departamento de Ciências da Vida, Universidade do Estado da Bahia (UNEB)

Rua Silveira Martins, 2555 - Cabula - Salvador, BA, Brasil - CEP 41150-000

E-mail: luanaleite_nutri@yahoo.com.br

\section{Submissão}

9 de maio de 2020

Aceito para publicação

30 de julho de 2020

\begin{abstract}
RESUMO
Introdução: A hidroclorotiazida é um dos fármacos utilizados para tratamento da hipertensão arterial sistêmica, componente da síndrome metabólica. $O$ uso desse medicamento está relacionado a perdas de nutrientes, como o magnésio, fato que favorece a deficiência nutricional para aqueles que apresentam o consumo alimentar inadequado desse mineral. Desta forma, este estudo teve como objetivo avaliar o consumo alimentar de magnésio em pacientes com síndrome metabólica, que fazem uso de hidroclorotiazida. Método: Trata-se de um estudo de delineamento transversal, analítico-descritivo, retrospectivo, realizado em uma clínica escola de nutrição em Salvador-BA, no qual foi feita a avaliação do consumo alimentar de magnésio de 35 pacientes, conforme os valores estabelecidos pela Dietary Reference Intake, e de acordo com o sexo, faixa etária, escolaridade, renda familiar e terapia com polifarmácia. A análise de dados foi realizada considerando significância estatística quando valor-p $\leq 0,05$. Resultados: A média do consumo alimentar de magnésio encontrada foi de 188,02 $\pm 93,9$ $\mathrm{mg}$ e o percentual de inadequação segundo a Estimated Average Requirement e Recommended Dietary Allowances foi de $80 \%$ e $85,7 \%$, respectivamente. O consumo alimentar médio revelou maiores resultados para: homens $(197,22 \pm 27,7 \mathrm{mg})$ em comparação às mulheres $(186,83 \pm 99,5 \mathrm{mg})(p=0,652)$; adultos de 20 a 39 anos $(195,69 \pm 139,6 \mathrm{mg})$ em comparação a outras faixas etárias ( $p=0,935)$; os que cursaram até ensino médio $(205,95 \pm 102,5 \mathrm{mg})$ em comparação a outras escolaridades $(p=0,490)$; os que tinham renda familiar de até 1 salário mínimo $(207,06 \pm 107,2 \mathrm{mg})$ em comparação a outras rendas $(p=0,640)$; e os que utilizavam polifarmácia $(204,08 \pm 104,4 \mathrm{mg})$ em comparação aos que não utilizavam $(174,50 \pm 84,5 \mathrm{mg})(p=0,371)$. Porém, esses achados não foram estatisticamente significativos e todos foram inferiores aos valores de referências estabelecidos. Conclusões: Os resultados encontrados revelaram um consumo alimentar inadequado de magnésio. Observa-se a necessidade da adoção de estratégias nutricionais para o incentivo ao consumo de alimentos fonte de magnésio para os pacientes com síndrome metabólica.
\end{abstract}

\section{ABSTRACT}

Introduction: Hydrochlorothiazide is one of the drugs used to treat systemic arterial hypertension, a component of metabolic syndrome. The use of this medication is related to nutrient losses such as magnesium, a fact that favors nutritional deficiency for those with inadequate food consumption of this mineral. Thus, this study aimed to assess food intake of magnesium in patients with metabolic syndrome, who use hydrochlorothiazide. Methods: This is a cross-sectional, analytical-descriptive, retrospective study, carried out in a nutrition school clinic in Salvador-BA, in which the evaluation of the magnesium food consumption of 35 patients was made, according to the values established by the Dietary Reference Intake, and according to sex, age group, education, family income and polypharmacy therapy. Data analysis was performed considering statistical significance when $p$-value $\leq 0.05$. Results: The average magnesium food consumption found was $188.02 \pm 93.9 \mathrm{mg}$ and the percentage of inadequacy according to Estimated Average Requirement and Recommended Dietary Allowances was $80 \%$ and $85.7 \%$, respectively. The average food consumption revealed higher results for: men (197.22 27.7 $\mathrm{mg})$ compared to women $(186.83 \pm 99.5 \mathrm{mg})(\mathrm{p}=0.652)$; adults aged 20 to 39 years (195.69 \pm 139.6 $\mathrm{mg}$ ) compared to other age groups $(\mathrm{p}=0.935)$; those who attended high school $(205.95 \pm 102.5 \mathrm{mg})$ compared to other schooling $(p=0.490)$; those who had a family income of up to 1 minimum wage $(207.06 \pm 107.2 \mathrm{mg})$ compared to other family incomes $(p=0.640)$; and those who used polypharmacy $(204.08 \pm 104.4 \mathrm{mg})$ compared to those who did not use it $(174.50 \pm 84.5 \mathrm{mg})(p=0.371)$. However, these findings were not statistically significative and all were below the established reference values. Conclusions: The results found revealed an inadequate food intake of magnesium. There is a need to adopt nutritional strategies to encourage the consumption of foods containing magnesium for patients with metabolic syndromic.

Nutricionista; Pós-graduanda em Nutrição Esportiva e Estética na Universidade Salvador (UNIFACS), Salvador, BA, Brasil.

2. Nutricionista; Mestre em Gestão e Tecnologia Aplicadas à Educação (GESTEC) pela Universidade do Estado da Bahia (UNEB); Docente do Curso de Bacharelado em Nutrição, Departamento de Ciências da Vida, Universidade do Estado da Bahia (UNEB), Salvador, BA, Brasil.

3. Nutricionista; Doutoranda e Mestre em Alimentos, Nutrição e Saúde pela Universidade Federal da Bahia (UFBA); Docente do Curso de Bacharelado em Nutrição, Departamento de Ciências da Vida, Universidade do Estado da Bahia (UNEB), Salvador, BA, Brasil.

4. Nutricionista; Doutora em Biotecnologia pela Universidade Estadual de Feira de Santana (UEFS)/FIOCRUZ; Docente do Curso de Bacharelado em Nutrição, Departamento de Ciências da Vida, Universidade do Estado da Bahia (UNEB), Salvador, BA, Brasil.

5. Biólogo; Doutor e Mestre em Patologia Humana pela Universidade Federal da Bahia (UFBA)/FIOCRUZ; Docente do Mestrado Profissional em Gestão e Tecnologias Aplicadas à Educação (GESTEC), Departamento de Educação, Universidade do Estado da Bahia (UNEB); Docente do Departamento de Ciências da Vida, Universidade do Estado da Bahia (UNEB), Salvador, BA, Brasil.

6. Médico; Pós-Doutor em Genética Cardiovascular pela University College London (UCL); Doutor em Genética e Biologia Molecular pela Universidade Federal do Rio Grande do Sul (UFRGS); Docente de Genética Humana, Departamento de Ciências da Vida, Universidade do Estado da Bahia (UNEB), Salvador, BA, Brasil. 


\section{INTRODUÇ̃̃O}

A síndrome metabólica (SM) envolve um conjunto de fatores de risco cardiovascular relacionados ao acúmulo abdominal de gordura e à resistência à insulina'. Estudos internacionais ${ }^{2,3}$ indicam taxas de prevalência de SM que variam entre $30,4 \%$ e $41 \%$. No Brasil, na faixa etária acima de 60 anos, essas taxas variam entre $37,2 \%$ e $49,1 \%$, em diferentes regiões ${ }^{4,5}$.

De acordo com a International Diabetes Federation ${ }^{6}$, para diagnóstico da SM, o indivíduo deve apresentar, obrigatoriamente, obesidade central e mais dois parâmetros dentre hiperglicemia, hipertrigliceridemia, baixos níveis do colesterol da lipoproteína de alta densidade (HDL-c) e hipertensão arterial sistêmica (HAS). Destas complicações, a HAS é um problema de saúde pública relevante, por ser a doença crônica não transmissível (DCNT) que mais acomete a população e o fator de risco primário no que se refere a doenças cardiovasculares e mortalidade ${ }^{7}$.

Os recursos terapêuticos utilizados para o controle da pressão arterial incluem tratamento não-farmacológico e o uso de medicamentos anti-hipertensivos com o objetivo de, além de reduzir a pressão arterial, preservar os órgãos-alvo, bem como evitar problemas cardiovasculares e renais ${ }^{8}$.

Referindo-se aos anti-hipertensivos, a hidroclorotiazida (HCTZ) é um medicamento pertencente à classe dos diuréticos tiazídicos, sendo que este fármaco, em conjunto com os betabloqueadores, foram os mais utilizados nos últimos 40 anos no tratamento de pacientes com HAS'. Os diuréticos tiazídicos conseguem regular a pressão arterial, reduzindo de forma antecipada o volume intravascular e a resistência vascular periférica, porque impossibilitam a reabsorção de sódio e cloreto nos túbulos renais ${ }^{10}$. Desta forma, a ação da HCTZ provoca alterações nas concentrações de nutrientes no organismo, como o aumento da excreção urinária destes e outros eletrólitos, especialmente o magnésio ${ }^{11}$.

Segundo Cruz-Aranda ${ }^{12}$, o tratamento por longos períodos com os diuréticos tiazídicos favorece a diminuição de cerca de $5 \%$ a $10 \%$ do magnésio plasmático. Este nutriente é o segundo cátion em maior quantidade no ambiente intracelular, sendo um elemento indispensável para manutenção dos processos celulares, participando de reações essenciais como no metabolismo dos macronutrientes e regulação dos sistemas hormonal e imunológico ${ }^{13}$.

Além disso, apresenta importante finalidade na regulação da pressão arterial devido a sua atividade como inibidor da contração da musculatura lisa vascular, podendo exercer uma função de vasodilatador ${ }^{14}$. Ainda contribui no desenvolvimento e manutenção dos ossos, participando da composição mineral óssea, em conjunto com o fosfato e cálcio, e auxiliando nas trocas que ocorre entre esses elementos e os ossos e tecidos ${ }^{15}$.
Dentre as manifestações clínicas observadas em casos de deficiências do magnésio se encontram as relacionadas ao sistema nervoso central (anorexia, náuseas e vômitos), do sistema neuromuscular (cãibras, fraqueza muscular e convulsões) e do sistema cardiovascular (trombose), além da predisposição à arritmia ${ }^{16}$.

Sendo assim, uma vez que o magnésio é um nutriente essencial ao organismo e sua deficiência pode trazer diversas consequências negativas para o indivíduo, principalmente as relacionadas ao sistema cardiovascular, cabe avaliar o consumo alimentar para detectar possíveis insuficiências na ingestão e incentivar o consumo de alimentos fontes deste nutriente. Neste contexto, o presente estudo teve como objetivo avaliar o consumo alimentar de magnésio em pacientes com síndrome metabólica, que fazem uso de hidroclorotiazida.

\section{MÉTODO}

Trata-se de um estudo de delineamento transversal, retrospectivo, de natureza quantitativa, com abordagem analítico-descritiva, realizado em uma clínica escola de nutrição do município de Salvador-BA. Os dados coletados foram de pacientes atendidos no período de abril de 2014 a abril de 2015 e oriundos do banco de dados do Núcleo de Pesquisa e Extensão em Genômica Nutricional e Disfunções Metabólicas (GENUT). Este estudo é um recorte do projeto de pesquisa intitulado "Influência de ações educacionais sobre as interações fármacos-nutrientes em pacientes portadores de síndrome metabólica", aprovado pelo Comitê de Ética em Pesquisa da Universidade do Estado da Bahia, sob o parecer n $733956 / 2014$.

Foram incluídos dados secundários de 35 pacientes, de ambos os sexos, adultos, com faixa etária acima de 20 anos, diagnosticados com HAS como componente da SM e que faziam uso de HCTZ e apresentaram dois recordatórios de 24 horas (R24hs) respondidos de maneira integral no prontuário. Não foram incluídos na pesquisa aqueles que faziam uso de suplementação de magnésio e os que apresentaram qualquer condição de saúde ou doença que poderia interferir na fidedignidade dos dados, como gestantes, lactantes, pacientes bariátricos, portadores de doenças degenerativas, doenças inflamatórias intestinais e insuficiência renal aguda e crônica.

Foram coletados dados demográficos, socioeconômicos e clínicos dos pacientes, e foi realizada a análise dos R24hs, a fim de caracterizar o consumo alimentar de magnésio. Para tal, foram utilizados dois recordatórios referentes à consulta inicial, sendo um de dia típico e o outro de dia atípico (final de semana), não consecutivos. Os cálculos dos recordatórios foram feitos com auxílio do software de Nutrição Nutrilife ${ }^{\circledR}$ e, em seguida, encontrou-se a média de consumo alimentar de magnésio de cada paciente. 
A classificação de adequação utilizada para o consumo médio foi de acordo com os valores estabelecidos pela Dietary Reference Intakes (DRI's) para a Ingestão Dietética Recomendada (Recommended Dietary Allowances - RDA) e a Necessidade Média Estimada (Estimated Average Requirement - EAR). Os valores de referência utilizados se encontram na Tabela 1.

Tabela 1 - Valores da Ingestão Dietética Recomendada (RDA) e da Necessidade Média Estimada (EAR) do magnésio, segundo sexo e faixa etária.

\begin{tabular}{lll}
\hline & EAR $(\mathrm{mg})$ & RDA $(\mathrm{mg})$ \\
\hline Mulheres & & \\
$20-30$ anos & $\geq 255$ & $\geq 310$ \\
$\geq 31$ anos & $\geq 265$ & $\geq 320$ \\
\hline Homens & $\geq 330$ & \\
$20-30$ anos & $\geq 350$ & $\geq 400$ \\
$\geq 31$ anos & &
\end{tabular}

Fonte: Adaptado de Institute of Medicine ${ }^{17}$.

As variáveis categóricas foram representadas a partir de frequências absoluta e relativa (\%) e as variáveis quantitativas, em média e desvio padrão, considerando a distribuição normal dos dados. Com relação ao perfil de consumo alimentar do magnésio, foi avaliado o percentual de adequação, além de relacionada a média diária de consumo aos determinantes sexo, faixa etária, escolaridade, renda familiar e terapia com polifarmácia (prática caracterizada pelo uso concomitante de cinco ou mais medicamentos por uma mesma pessoa ${ }^{18}$ ).

A análise estatística foi realizada no programa SPSS Statistics para Windows, versão 20.0.0., utilizando-se o teste estatístico ANOVA e teste † de Student para diferença de médias, considerando significância estatística quando valor-p $\leq 0,05$.

\section{RESULTADOS}

A caracterização da população estudada encontra-se na Tabela 2. A amostra foi composta de 35 pacientes, com predomínio do sexo feminino $(88,6 \%)$. A média de idade dos participantes foi de $54,91 \pm 8,3$ anos e observou-se que $68,6 \%$ dos indivíduos se encontravam na faixa etária de 40 a 59 anos.

Em relação à escolaridade, a mesma quantidade de pacientes que cursou o ensino fundamental $(45,7 \%)$, cursou o ensino médio (45,7\%). Quanto à renda familiar, houve predomínio daqueles que recebiam até 1 salário mínimo $(42,9 \%)$, seguidos pela renda de 1 a 2 salários mínimos $(37,1 \%)$. Já no que diz respeito à terapia medicamentosa com polifarmácia, $45,7 \%$ faziam uso de cinco ou mais medicamentos.
Tabela 2 - Caracterização segundo variáveis demográficas, socioeconômicas e clínicas de pacientes com síndrome metabólica que fazem uso de hidroclorotiazida. Salvador-BA, 2020.

\begin{tabular}{lcc}
\hline Variável & N & $\%$ \\
\hline Sexo & 31 & 88,6 \\
$\quad$ Feminino & 4 & 11,4 \\
$\quad$ Masculino & & \\
\hline Faixa etária & 3 & 8,6 \\
20 a 39 anos & 24 & 68,6 \\
40 a 59 anos & 8 & 22,9 \\
$\geq 60$ anos & & \\
\hline Escolaridade & 1 & 2,9 \\
Analfabeto & 16 & 45,7 \\
Ensino fundamental & 16 & 45,7 \\
Ensino médio & 2 & 5,7 \\
Ensino superior & & \\
Renda familiar (em salários mínimos) & 15 & 42,9 \\
Até 1 & 13 & 37,1 \\
1 a 2 & 5 & 14,3 \\
2 a 3 & 2 & 5,7 \\
3 a 4 & & 45,7 \\
\hline Terapia com polifarmácia & 16 & 54,3 \\
Sim & 19 & \\
Não & & \\
\hline & & \\
\hline
\end{tabular}

Os dados referentes à adequação do consumo alimentar de magnésio, utilizando os parâmetros da DRI's, estão presentes na Tabela 3. Foi observado que ao avaliar pela EAR, $80 \%$ dos pacientes apresentavam consumo médio diário de magnésio abaixo dos valores de referência e ao utilizar a RDA como indicador, esse valor foi de $85,7 \%$. A média geral de consumo foi de 188,02 $\pm 93,9 \mathrm{mg}$, valor abaixo das recomendações, independente do indicador utilizado.

Tabela 3 - Adequação do consumo alimentar de magnésio dos pacientes com síndrome metabólica que fazem uso de hidroclorotiazida. Salvador-BA, 2020.

\begin{tabular}{lcccc}
\hline & $\begin{array}{c}\text { < EAR } \\
\% \text { (n) }\end{array}$ & $\begin{array}{c}\geq \text { EAR e } \\
<\text { RDA } \\
\% \text { (n) }\end{array}$ & $\begin{array}{c}<\text { RDA } \\
\%(\mathbf{n})\end{array}$ & $\begin{array}{c}\geq \text { RDA } \\
\%(\mathbf{n})\end{array}$ \\
\hline $\begin{array}{l}\text { Consumo alimentar } \\
\text { de magnésio }\end{array}$ & $80(28)$ & $5,7(2)$ & $85,7(30)$ & $14,3(5)$ \\
\hline EAR = Necessidade Média Estimada; RDA = Ingestão Dietética Recomendada.
\end{tabular}

Na Tabela 4, encontram-se os dados referentes ao consumo alimentar de magnésio, conforme características demográficas, socioeconômicas e clínicas dos pacientes. Os resultados identificados não apresentaram significância estatística. 
Tabela 4 - Consumo alimentar de magnésio (mg) e aspectos demográficos, socioeconômicos e clínicos dos pacientes com síndrome metabólica que fazem uso de hidroclorotiazida. Salvador-BA, 2020.

\begin{tabular}{lcc}
\hline Variável & \multicolumn{2}{c}{ Consumo de magnésio } \\
\cline { 2 - 3 } Associada & $\begin{array}{c}\text { Média } \pm \text { desvio } \\
\text { padrão }(\mathbf{m g})\end{array}$ & Valor de $p$ \\
\hline Gênero & & \\
Feminino & $186,83 \pm 99,5$ & $\mathrm{p}=0,652^{*}$ \\
Masculino & $197,22 \pm 27,7$ & \\
\hline Faixa etária & & \\
20 a 39 anos & $195,69 \pm 139,6$ & $\mathrm{p}=0,935^{\star *}$ \\
40 a 59 anos & $190,64 \pm 93,1$ & \\
$\geq 60$ anos & $177,28 \pm 92,8$ & \\
\hline Escolaridade & & \\
Analfabetos & & \\
Ensino fundamental & $180,48 \pm 94,0$ & \\
Ensino médio & $205,95 \pm 102,5$ & \\
Ensino superior & $112,17 \pm 42,1$ & \\
\hline Renda familiar (em salários mínimos) & \\
Até 1 & $207,06 \pm 107,2$ & $\mathrm{p}=0,640^{\star *}$ \\
1 a 2 & $162,60 \pm 83,0$ & \\
2 a 3 & $203,79 \pm 98,6$ & \\
3 a 4 & $171,06 \pm 41,1$ & \\
\hline
\end{tabular}

\section{Terapia com polifarmácia}

$\begin{array}{lc}\text { Sim } & 204,08 \pm 104,4 \quad p=0,371^{*} \\ \text { Não } & 174,50 \pm 84,5\end{array}$

* Teste $t$ de Student, valor de $p \leq 0,05$. ${ }^{* *}$ Teste ANOVA, valor de $p \leq 0,05 .(-)=$ ausentes; (mg=miligrama).

Ao avaliar o consumo alimentar de acordo com o sexo dos participantes, observou-se que a média diária foi maior no sexo masculino $(197,2 \pm 27,7 \mathrm{mg})$ em comparação ao sexo feminino $(186,8 \pm 99,5 \mathrm{mg})(\mathrm{p}=0,652)$.

Tratando-se da faixa etária, a média diária de consumo de magnésio foi maior na faixa etária 20 a 39 anos (195,69 \pm $139,6 \mathrm{mg})$ e menor na faixa etária acima e 60 anos $(177,28$ $\pm 92,8 \mathrm{mg})(p=0,935)$. No que diz respeito à escolaridade, foi revelada uma média diária de consumo maior para os que cursaram até o ensino médio $(205,95 \pm 102,5 \mathrm{mg})$ e menor média diária para os que cursaram o ensino superior $(112,17 \pm 42,1 \mathrm{mg})(\mathrm{p}=0,490)$.

Também foi observado que o consumo desse nutriente foi maior para aquelas pessoas que tinham a renda familiar de até 1 salário mínimo, apresentando a média de 207,06 \pm 107,2 mg, e menor na renda de 1 a 2 salários mínimos com média de 162,60 $\pm 83,0 \mathrm{mg}(\mathrm{p}=0,640)$. Em relação à polifarmácia, os pacientes que faziam uso dessa terapia apresentaram uma média diária mais elevada $(204,08 \pm 104,4 \mathrm{mg})$ do que os que não faziam $(174,50 \pm 84,5 \mathrm{mg})(\mathrm{p}=0,371)$.

\section{DISCUSSÃO}

O presente estudo avaliou o consumo alimentar de magnésio em pacientes com síndrome metabólica em uso de hidroclorotiazida, identificando ingestão inadequada desse nutriente de acordo com os parâmetros propostos pelas DRI's (RDA e EAR) e conforme as médias diárias de consumo, revelando, portanto, a necessidade da devida atenção e de maior direcionamento no manejo nutricional deste público.

A maioria dos participantes apresentou consumo inadequado de magnésio, abaixo dos valores de referência, assim como foi verificado por Jackson et al. ${ }^{19}$, em uma pesquisa em que foi avaliada a associação entre etnia e ingestão de magnésio de 5.682 idosos americanos, na qual 83,3\% da população apresentaram inadequação de consumo segundo a RDA.

Essas prevalências de inadequação identificadas são semelhantes às encontradas em um estudo do consumo alimentar de 96 usuários de uma Clínica Escola de Nutrição no município de Americana-SP, no qual 88,54\% da amostra apresentavam ingestão abaixo da EAR e $92,7 \%$ a ingestão menor ou igual a RDA ${ }^{20}$. Nesta mesma pesquisa, também foi avaliada, no grupo de pacientes que apresentavam DCNT, a média diária de consumo de magnésio que foi de 228,06 \pm 151,17 mg, valor abaixo das recomendações, assim como no atual estudo.

Serban et al. ${ }^{21}$ realizaram uma pesquisa com 409 adultos obesos que estavam sob supervisão médica, em que foi avaliada a prevalência das doses recomendadas de macro e micronutrientes, e os participantes também apresentaram o consumo médio de magnésio abaixo das recomendações, com o valor de 225,8 $\pm 104,3 \mathrm{mg} /$ dia. Resultado semelhante foi encontrado em um estudo realizado com o objetivo de investigar as associações entre os valores basais de ingestão de magnésio e sintomas do sono, em que a mediana de consumo de 1.487 participantes no baseline foi $289 \mathrm{mg} / \mathrm{dia}$ (intervalo interquartil 233,7-366,1 mg/dia) ${ }^{22}$.

Estes achados podem estar relacionados à ingestão reduzida de alimentos fontes de magnésio, como os vegetais folhosos verdes, cereais integrais, frutas, legumes e tubérculos $^{23}$. É possível identificar que muitos alimentos cultivados de forma natural possuem este nutriente em sua composição, mas, nas últimas décadas, seu consumo reduziu de maneira significativa devido a variados fatores, entre eles, alterações em hábitos alimentares ${ }^{24}$. No Brasil, este fato pode ser observado, já que houve mudanças no sistema alimentar do país, em que foi identificado um aumento na disponibilidade e consumo de alimentos processados e ultraprocessados em detrimento do consumo de alimentos in natura e minimamente processados ${ }^{25}$.

Este fenômeno afeta negativamente a quantidade de micronutrientes na dieta e considerando que a população 
do presente estudo já se encontra em risco nutricional devido às doenças de base e que a hidroclorotiazida normalmente é prescrita para uso contínuo, é importante salientar que o consumo inadequado do magnésio favorece ainda mais ao desenvolvimento de um quadro de deficiência nutricional ${ }^{16}$.

Embora os mecanismos não estejam completamente elucidados, a hipomagnesemia pode gerar consequências relacionadas à homeostase da glicose e à hipertensão arterial. Rosique-Esteban et al. ${ }^{26}$ afirmam que baixos níveis de magnésio podem intensificar os processos associados à resistência à insulina, por causar alterações, como a diminuição na secreção pancreática deste hormônio, e interferir nas interações insulina-receptor. Assim como, acredita-se que o consumo inadequado de magnésio ou interferências no seu metabolismo pode promover vasoespasmo ou dano ao endotélio, afetando a pressão arterial e a função endotelial.

O magnésio também atua no metabolismo cerebral, desta forma, sua deficiência está associada a sinais e sintomas neuromusculares e psiquiátricos, como enxaqueca, fraqueza muscular, ansiedade, insônia e depressão. Além disso, a hipomagnesemia está relacionada a outros distúrbios, como a insuficiência cardíaca congestiva e arritmias, sendo que algumas pesquisas apontam que a suplementação deste nutriente auxilia no tratamento dessas e outras doenças ${ }^{27}$.

No presente estudo, apesar de não ser estatisticamente significativo, o sexo masculino apresentou maior adequação no consumo de magnésio, como encontrado por Souza et al. ${ }^{28}$ em uma pesquisa para avaliar a ingestão de nutrientes de 60 pacientes com neurofibromatose tipo 1, na qual os homens apresentaram média de consumo mais elevada que as mulheres, $219,6 \mathrm{mg}$ (mínimo $=95,4 \mathrm{mg}$ e máximo $=$ 381,4 mg) e 166,7 mg (mínimo $=77,3$ mg e máximo $=$ 280,0 mg), respectivamente, não apresentando significância estatística ( $p=0,641)$. Resultado semelhante ao de um estudo ${ }^{29}$ realizado para avaliar a associação de ingestão de minerais e vitaminas do complexo B sobre níveis de glicemia, em que a média diária de consumo foi maior no sexo masculino $(372,71$ mg - intervalo de confiança 95\%: 362,48-382,94 mg) que no feminino (305,34 mg - intervalo de confiança 95\%: 294,20$316,48 \mathrm{mg}$ ). Assim como identificado por Venturini et al. ${ }^{30}$, em uma pesquisa sobre perfil de consumo de nutrientes e sua associação com as variáveis sociodemográficas e de saúde de 427 idosos em que os homens $(261,3 \pm 106,8 \mathrm{mg}$ ) apresentaram média de consumo mais elevada que as mulheres $(227,5 \pm 88,5 \mathrm{mg})$, contudo essa diferença foi estatisticamente significativa $(p=0,002)$.

Os achados deste estudo e dos citados corroboram com as recomendações preconizadas pela DRI's, nas quais os valores de referência de consumo de magnésio para os homens são mais elevados que para as mulheres. Além disso, a ingestão de magnésio normalmente é proporcional à ingestão calórica total, fato que justifica maiores níveis de consumo deste mineral para jovens e homens que consomem mais calorias que as mulheres e idosos ${ }^{17}$. Entretanto, ainda assim, todos resultados citados em relação à adequação de consumo no sexo masculino se encontram abaixo das recomendações estabelecidas.

Ferreira-Nunes et al. ${ }^{31}$ afirmam que, com o envelhecimento torna-se mais comum que fatores relacionados à redução da ingestão dos alimentos e aproveitamento dos nutrientes interfiram no estado nutricional das pessoas. No presente estudo, observou-se que a ingestão de magnésio foi inversamente relacionada à idade, ou seja, foi menor na senescência. Este achado pode estar relacionado, principalmente, a aspectos intrínsecos do envelhecimento que alteram os hábitos alimentares dos idosos, como a disfunção na percepção do paladar e alterações nos processos de mastigação e deglutição ${ }^{32}$.

As deficiências de micronutrientes que acometem a população de idade mais avançada, por estarem relacionadas a alterações funcionais, causam uma preocupação considerável em aspectos como autonomia e longevidade. O magnésio, nesse contexto, tem grande relevância devido a sua participação tanto no desenvolvimento de ossos saudáveis, quanto na formação e funcionalidade dos músculos nos idosos ${ }^{33}$.

Tratando-se da escolaridade, acredita-se que a educação escolar influencia no que diz respeito aos conhecimentos sobre alimentação e nutrição, consequentemente, interferindo no estado nutricional dos indivíduos ${ }^{34}$. Em um estudo realizado com idosos usuários das unidades básicas de saúde de Goiânia-GO, avaliou-se a prevalência do consumo diário de frutas, verduras e legumes. Os resultados revelaram que, quanto mais elevada a escolaridade, maior o consumo destes alimentos, sendo que estes são algumas das principais fontes de magnésio ${ }^{35}$.

Contudo, os dados desta pesquisa revelaram menores taxas de ingestão no ensino superior, podendo esta ser uma característica específica deste público ou desse estudo, especialmente porque um número reduzido de pacientes apresentava maior nível de escolaridade. Além disso, uma quantidade significativa de participantes cursou até o ensino médio, segunda maior categoria com mais anos de estudos descrita nesta pesquisa, e esta foi a que apresentou maior média de consumo de magnésio.

A renda familiar também é um fator muito importante para o desenvolvimento de hábitos alimentares saudáveis. Um maior nível de escolaridade e uma renda familiar mais elevada contribuem para uma alimentação nutricionalmente mais rica ${ }^{30}$. Todavia, neste estudo, a maior taxa de consumo de magnésio foi na renda familiar dos que recebem até 1 salário mínimo, podendo ser um indicativo que, apesar 
desse fator influenciar, ele pode não ser decisivo para uma boa alimentação, que pode depender, além do custo dos alimentos, de aspectos como disponibilidade e acesso ${ }^{36}$.

Uma alternativa que pode facilitar o acesso de toda a comunidade a alimentos como as frutas e outros vegetais é a implementação de hortas comunitárias, que estimula a própria população a cultivar estes alimentos, fontes de diferentes nutrientes com o baixo custo $^{37}$. Portanto, estes resultados reforçam o papel do nutricionista como agente de educação alimentar e nutricional, sendo uma de suas responsabilidades estabelecer estratégias que alcancem qualquer nível de escolaridade e renda. Além disso, cabe a estes profissionais buscar meios de orientar os pacientes sobre uma alimentação de qualidade, segura e de acordo com sua renda familiar.

Já com relação ao tratamento com polifarmácia, uma parcela significativa dos pacientes fazia uso dessa terapêutica e estes apresentaram consumo de magnésio mais adequado do que aqueles que utilizavam quatro medicamentos ou menos. Este talvez seja um fator de proteção, tendo em vista que indivíduos que apresentam muitas enfermidades ou fazem uso de diversos fármacos simultaneamente estão mais propícios a sofrerem efeitos de interações medicamentosas com uma repercussão indesejada ${ }^{38}$. Especialmente, considerando que na polifarmácia é comum o uso contínuo de medicamentos e que as interações entre fármacos e nutrientes podem, além de causar reações adversas, interferir no estado nutricional do indivíduo ${ }^{39}$.

Apesar da importância dos achados, este estudo tem algumas limitações, por ser transversal e retrospectivo. Sabe-se que análises transversais são limitantes para identificar relações causais e para investigar desfechos em um único momento no tempo ${ }^{40}$. Quanto aos estudos retrospectivos, por usarem dados existentes, uma das dificuldades está nas possíveis variações dos registros de dados, principalmente quando estes não são projetados para pesquisa, e sim com propósitos clínicos ou administrativos ${ }^{41}$.

Deve-se, também, considerar que o tamanho amostral poderia ter sido maior, possibilitando, então, a realização de análises inferenciais estatísticas. Além disso, destacase a falta de estudos sobre a relação entre a ingestão de magnésio e dados demográficos e socioeconômicos para comparação de resultados, fazendo-se necessária, então, a elaboração de mais pesquisas que elucidem essas relações. No entanto, essas limitações não comprometem a qualidade deste trabalho e nem as observações encontradas.

\section{CONCLUSÃO}

A média de consumo de magnésio dos pacientes encontrase abaixo das recomendações nutricionais, independente do parâmetro utilizado. Maiores médias do consumo alimentar de magnésio foram identificadas no sexo masculino, faixa etária de 20 a 39 anos, ensino médio, renda familiar de 1 a 2 salários mínimos e terapia medicamentosa com polifarmácia.

Sugere-se que novos estudos sejam realizados, investigando a relação entre consumo de magnésio e depleção deste micronutriente por interação com fármacos, como a hidroclorotiazida, especialmente em pacientes com doenças crônicas como SM, além do estímulo à adoção de estratégias nutricionais voltadas para o incentivo ao consumo de alimentos fontes deste nutriente.

\section{REFERÊNCIAS}

1. Sociedade Brasileira de Cardiologia, Sociedade Brasileira de Hipertensão, Sociedade Brasileira de Endocrinologia e Metabologia, Sociedade Brasileira de Diabetes, Associação Brasileira para Estudos da Obesidade. I Diretriz Brasileira de Diagnóstico e Tratamento da Síndrome Metabólica. Arq Bras Cardiol. 2005;84(Supl 1):3-28.

2. Farmanfarma KK, Kaykhaei MA, Adineh HA, Mohammadi M, Dabiri S, Ansari-Moghaddam A. Prevalence of metabolic syndrome in Iran: a meta-analysis of 69 studies. Diabetes Metab Syndr. 2019;13(1):792-9.

3. Gutiérrez-Solis AL, Banik SD, Méndez-González RM. Prevalence of metabolic syndrome in Mexico: a systematic review and meta-analysis. Metab Syndr Relat Disord. 2018;16(8):395-405.

4. Zoraski H, Fiametti M, Santos R, Gregoletto MLO, Cremonese C. Síndrome metabólica em idosos de Nova Roma do Sul, RS: prevalência e fatores associados. ABCS Health Sci. 2017;42(3):147-55.

5. Neves CVB, Mambrini JVM, Torres KCL, Teixeira-Carvalho A, Martins-Filho OA, Lima-Costa MF, et al. Associação entre síndrome metabólica e marcadores inflamatórios em idosos residentes na comunidade. Cad Saúde Pública. 2019;35(3):e00129918.

6. Albert SG, Zimmet P, Shaw J, Grundy SM. Metabolic syndrome. The IDF consensus worldwide definition of the metabolic syndrome. Brussels: International Diabetes Federation; 2006.

7. Moraes NS, Souza JAG, Miranda RD. Hipertensão arterial, diabetes mellitus e síndrome metabólica: do conceito à terapêutica. Rev Bras Hipertens. 2013;20(3):109-16.

8. Malachias MVB, Souza WKSB, Plavnik FL, Rodrigues CIS, Brandão AA, Neves MFT, et al. $7^{\text {a }}$ diretriz brasileira de hipertensão arterial. Arq Bras Cardiol. 2016;107(3 Supl 3):1-83.

9. Ribeiro CC, Andrade GMM, Couto RO. Estudos de equivalência farmacêutica e perfil de dissolução comparativo de medicamentos contendo hidroclorotiazida. Infarma Rev Ciênc Farm. 2018;30(1):5-13.

10. Kaiser EA, Lotze U, Schäfer HH. Increasing complexity: which drug class to choose for treatment of hypertension in the elderly? Clin Interv Aging. 2014;9:459-75.

11. Barcelos AC, Trein AM, Sousa GS, Fleury Neto L, Baldaça L. Efeitos cardiotóxicos resultantes da interação da risperidona com diuréticos tiazídicos. J Bras Psiquiatr. 2014;63(4):379-83.

12. Cruz-Aranda JE. Fármacos diuréticos: alteraciones metabólicas y cardiovasculares en el adulto mayor. Med Interna Méx. 2018;34(4):566-73.

13. Sampaio FA, Cruz KJC, Oliveira ARS, Marreiro DN. Influência da hipomagnesemia sobre a homeostase do ferro e estresse oxidativo no diabetes mellitus tipo 2. Nutrire. 2015;40(2):214-25.

14. Oliveira EP, Camargo KF, Castanho GKF, Nicola M, PorteroMcLellan KC, Burini RC. A variedade da dieta é fator protetor para a pressão arterial sistólica elevada. Arq Bras Cardiol. 2012;98(4):338-43. 
15. Premaor MO, Brondani JE. Nutrição e saúde óssea: a importância do cálcio, fósforo, magnésio e proteínas. Rev AMRIGS. 2016;60(3):253-63.

16. Monteiro TH, Vannuchi H. Funções plenamente reconhecidas de nutrientes: magnésio. Série de Publicações ILSI Brasil. Força-tarefa Alimentos Fortificados e Suplementos. Comitê de Nutrição ILSI Brasil. São Paulo: ILSI Brasil- International Life Sciences Institute do Brasil; 2010.

17. Institute of Medicine (US) Standing Committee on the Scientific Evaluation of Dietary Reference Intakes. Dietary reference intakes for calcium, phosphorus, magnesium, vitamin D, and fluoride. Washington: National Academy Press; 1997.

18. Silveira EA, Dalastra L, Pagotto V. Polifarmácia, doenças crônicas e marcadores nutricionais em idosos. Rev Bras Epidemiol. 2014;17(4):818-29.

19. Jackson SE, Smith L, Grabovac I, Haider S, Demurtas J, LópezSánchez GF, et al. Ethnic differences in magnesium intake in U.S. older adults: findings from NHANES 2005-2016. Nutrients. 2018;10(12):1901.

20. Marinho CF, Beltrame MAS, Ferreira KK, Nobre JAS, Córdoba GMC. Consumo alimentar de usuários de uma clínica-escola de nutrição do interior paulista. RASBRAN. 2017;8(1):52-7.

21. Serban CL, Sima A, Hogea CM, Chirită-Emandi A, Perva IT, Vlad A, et al. Assessment of nutritional intakes in individuals with obesity under medical supervision: a cross-sectional study. Int J Environ Res Public Health. 2019;16(17):3036.

22. Cao Y, Zhen S, Taylor AW, Appleton S, Atlantis E, Shi Z. Magnesium intake and sleep disorder symptoms: findings from the Jiangsu Nutrition Study of Chinese Adults at five-year followup. Nutrients. 2018;10(10):1354.

23. Severo JS, Morais JBS, Freitas TEC, Cruz KJC, Oliveira ARS, Poltronieri F, et al. Aspectos metabólicos e nutricionais do magnésio. Nutr Clín Diet Hosp. 2015;35(2):67-74.

24. Razzaque MS. Magnesium: are we consuming enough? Nutrients. 2018;10(12):1863.

25. Instituto Brasileiro de Geografia e Estatística. Pesquisa de orçamentos familiares 2017-2018 - POF. Rio de Janeiro: IBGE; 2020.

26. Rosique-Esteban N, Guasch-Ferré M, Hernández-Alonso P, Salas-Salvadó J. Dietary magnesium and cardiovascular disease: a review with emphasis in epidemiological studies. Nutrients. 2018;10(2):168.

27. Ramirez AVG. A importância do magnésio na doença cardiovascular. Intern J Nutrol. 2016;9(4):242-53.

28. Souza ML, Jansen AK, Martins AS, Rodrigues LO, Rezende NA. Nutrient intake in neurofibromatosis type 1: a cross-sectional study. Nutrition. 2015;31(6):858-62.
29. Yan S, Li M, Ma X, Jiang S, Sun M, Wang C, et al. Association of multiple mineral and vitamin B group intake with blood glucose using quantile regression analysis: NHANES 2007-2014. Food Nutr Res. 2019;63.

30. Venturini CD, Engroff P, Sgnaolin V, El Kik RM, Morrone FB, Silva Filho IG, et al. Consumo de nutrientes em idosos residentes em Porto Alegre (RS), Brasil: um estudo de base populacional. Ciênc Saúde Coletiva. 2015;20(12):3701-11.

31. Ferreira-Nunes PM, Papini SJ, Corrente JE. Padrões alimentares e ingestão de nutrientes em idosos: análise com diferentes abordagens metodológicas. Ciênc Saúde Coletiva. 2018;23(12):4085-94.

32. Monteiro MAM, Maia ICMP. Perfil alimentar de idosos em uma instituição de longa permanência de Belo Horizonte, MG. Rev APS. 2015;18(2):199-204.

33. Ter Borg S, Verlaan S, Hemsworth J, Mijnarends DM, Schols JMGA, Luiking YC, et al. Micronutrient intakes and potential inadequacies of community-dwelling older adults: a systematic review. Br J Nutr. 2015;113(8):1195-206.

34. Moura AF, Masquio DCL. A influência da escolaridade na percepção sobre alimentos considerados saudáveis. Rev Ed Popular. 2014;13(1):82-94.

35. Silveira EA, Martins BB, Abreu LRS, Cardoso CKS. Baixo consumo de frutas, verduras e legumes: fatores associados em idosos em capital no Centro-Oeste do Brasil. Ciênc Saúde Coletiva. 2015;20(12):3689-99.

36. Berti TL, Rocha TF, Curioni CC, Verly Junior E, Bezerra FF, Canella DS, et al. Consumo alimentar segundo o grau de processamento e características sociodemográficas: estudo Pró-Saúde. Rev Bras Epidemiol. 2019;22:E190046.

37. Costa CGA, Garcia MT, Ribeiro SM, Salandini MFS, Bógus CM. Hortas comunitárias como atividade promotora de saúde: uma experiência em Unidades Básicas de Saúde. Ciênc \& Saúde Colet. 2015;20(10):3099-110.

38. Peixoto JS, Salci MA, Radovanovic CAT, Salci TP, Torres MM, Carreira L. Riscos da interação droga-nutriente em idosos de instituição de longa permanência. Rev Gaúcha Enferm. 2012;33(3):156-64.

39. Carlos GB, Francisco LN, Moraes TC, Cerdeira CD, Santos GB. Análise das possíveis interações fármaco-alimento/nutriente em uma instituição asilar no sul de Minas Gerais. Rev Bras Pesq Saúde. 2016;18(3):83-90.

40. Rothman KJ, Greenland S, Lash TL. Types of epidemiological studies. In: Rothman KJ, Greenland S, Lash T, eds. Modern epidemiology. $3^{\text {rd }}$ ed. Porto Alegre: Artmed; 2011. p.107-22.

41. Sessler DI, Imrey PB. Clinical research methodology 1: study designs and methodologic sources of error. Anesth Analg. 2015;121(4):1034-42.

Local de realização do estudo: Centro de Estudos e Atendimento Dietoterápico (CEAD), Departamento de Ciências da Vida, Universidade do Estado da Bahia (UNEB), Salvador, BA, Brasil.

Conflito de interesse: Os autores declaram não haver. 